\title{
Predictors of withdrawal of life support after burn injury
}

\author{
Colleen N. Bartley, Kenisha Atwell, Bruce Cairns, Anthony Charles* \\ Department of Surgery, University of North Carolina at Chapel Hill, North Carolina Jaycee Burn Center, United States
}

A R T I C L E I N F O

Article history:

Accepted 15 October 2018

Keywords:

Burn injury

Withdrawal of life support

End of life decisions

Comfort care

\begin{abstract}
A B S T R A C T
Introduction: Discussions regarding withdrawal of life support after burn injury are challenging and complex. Often, providers may facilitate this discussion when the extent of injury makes survival highly unlikely or when the patient's condition deteriorates during resuscitation. Few papers have evaluated withdrawal of life support in burn patients. We therefore sought to determine the predictor of withdrawal of life support (WLS) in a regional burn center.

Methods: We conducted a retrospective analysis of all burn patients from 2002 to 2012. Patient characteristics included age, gender, burn mechanism, percentage total body surface area (\% TBSA) burned, presence of inhalation injury, hospital length of stay, and pre-existing comorbidities. Patients <17years of age and patients with unknown disposition were excluded. Patients were categorized into three cohorts: Alive till discharge (Alive), death by withdrawal of life support (WLS), or death despite ongoing life support (DLS). DLS patients were then excluded from the study population. Multivariate logistic regression was used to estimate predictors of WLS.

Results: 8,371 patients were included for analysis: 8134 Alive, 237 WLS. Females had an increased odd of WLS compared to males (OR 2.03, 95\% CI 1.18-3.48; $p=0.010$ ). Based on higher CCI, patients with pre-existing comorbidities had an increased odd of WLS (OR 1.28, $95 \%$ CI 1.08-1.52; $\mathrm{p}=0.005$ ). There was a significantly increased odds for WLS (OR 1.09, 95\% CI 1.06-1.12; $\mathrm{p}<0.001)$ with increasing age. Similarly, there was an increased odd for WLS (OR 1.08, 95\% CI 1.07-1.51; $\mathrm{p}<0.001$ ) with increasing \%TBSA. An increased odd of WLS (OR $2.47,95 \%$ CI 1.05-5.78; $p=0.038$ ) was also found in patients with inhalation injury.

Conclusion: The decision to withdraw life support is a complex and difficult decision. Our current understanding of predictors of withdrawal of life support suggests that they mirror those factors which increase a patient's risk of mortality. Further research is needed to fully explore end-of-life decision making in regards to burn patients. The role of patient's sex, particularly women, in WLS decision making needs to be further explored.
\end{abstract}

\section{Introduction}

According to the American Burn Association, over 480,000 patients receive hospital and emergency room treatment for burns annually [1]. It is estimated that there were 3390 fire related civilian deaths in the United States in 2016, the highest number recorded since 2008 [2]. Many of these deaths will occur in the hospital following a decision to withhold or withdraw life sustaining treatment.

\footnotetext{
* Corresponding author at: Associate Professor of Surgery, UNC School of Medicine, 4008 Burnett Womack Building, CB 7228, United States. E-mail address: anthchar@med.unc.edu (A. Charles).

https://doi.org/10.1016/j.burns.2018.10.015
} 
The decision to withdraw life support is a difficult and complex one. The process is often initiated when a patient's injury is deemed non-survivable or when ongoing treatment is deemed futile [3]. A combination of objective and subjective criteria, including extent and depth of injury, pre-existing medical comorbidities (especially in the older population), clinician knowledge and previous experiences, potential future quality of life, and likelihood to return to independent living are considered [3-5]. Patient and family input is important, and necessary, in this decision-making process as the ultimate goal is to act in accordance with patient wishes.

Previous studies have evaluated the timing of withdrawal of life support. For children this decision occurs late, hours before death [6]. In adults, the definition of "Early" vs "Late" withdrawal varies. Nevertheless, the majority of patient deaths have been shown to occur following withdrawal of life support $[3,7]$.

With advances in modern burn care, we are able to provide increasingly complex care, allowing for improved survival in patients who decades ago would have died. This has not been without consequence to our health care system. Burn care delivery is considered one of the most expensive within the current health care system in the United States due to critical care costs $[8,9]$. It has been suggested that the goal of burn care should shift from saving lives to making sure that those patients who survive their injuries are able to return to productive lives [21].

The determinants of burn mortality have been well documented. Classically they include age, burn size, and presence of inhalation injury and comorbidities [10-13]. Additionally, there are a number of predictive models which exist to assist clinicians in determining survival [14-18]. While end-of-life care for critically ill patients has been well researched [19-22], there remains a paucity of information regarding end of life decision making in the burn population.

Objective predictors of death following burn injury help clinicians make decisions and reduce reliance on clinical intuition [23]. The goal of this paper was to evaluate burn patients admitted to our burn intensive care unit (ICU) and provide potential objective predictors of withdrawal of life support.

\section{Methods}

This is a retrospective analysis of burn patients admitted to the North Carolina Jaycee Burn Center at the University of North Carolina (UNC) from 2002 to 2012. The Jaycee Burn Center was established in 1981 and averages more than 1,600 acute admissions per year. The burn center is a single unit, 36-ed facility that has been verified by the American Burn Association for pediatric and adult care. Approval for the study was obtained from the UNC Institutional Review Board.

The medical records of subjects identified by the UNC Burn database query were reviewed to verify baseline demographic data, injury characteristics, and provide detailed information on medical comorbidities. Pre-existing comorbidities were obtained from medical records and recorded into the burn registry. This information is reported from patient, family, or others that intimately know the patient's history upon admission to the burn center. Injury characteristic of interest included burn etiology, \% total body surface are (\%TBSA) burn, presences of inhalation injury, and whether or not a patient was instituted during admission to the burn center. Inhalation injury diagnosis was based on history, physical examination, and/or bronchoscopic examination. All patients in the ICU were treated with the prevailing standard of care (i.e. ventilator management, fluid resuscitation, etc.) at a large academic burn center at the time of admission.

All patients $\geq 17$ years of age who were admitted during the study period were included in this analysis. Patients were excluded if missing discharge disposition. Patients were then placed into one of three cohorts: Alive till discharge (Alive), death by withdrawal of life support (WLS), or death despite ongoing life support (DLS). WLS was defined as those patients whose discharge summary reported withdrawal of care, withdrawal of life sustaining support, and comfort care measures were instituted. Those patients in the DLS group were then excluded from further analysis in order to better elucidate risk factors for withdrawal of life support. Age was further dichotomized $<65$ and $\geq 65$ years to distinguish potential age-related factors associated with the decision to withdraw care. Race was categorized into White, Black, Hispanic, and other. Burn etiology was categorized into Flame, Scald, and Other (all other types of burns including contact, chemical, electrical, or radiation burns). A Charlson Comorbidity Index (CCI) score was calculated for each patient and used to examine the effect of preexisting medical comorbidities on outcome [24]. The CCI score is the weighted sum of comorbid conditions. There are 17 comorbid conditions included in the score and each is assigned a weight from one to six points. The weighted sum of all comorbid conditions is the patient's Charlson score.

WLS patients were further categorized into Early WLS and Late WLS. Early WLS patients were those for whom a decision was made to withdraw life support within the first $24 \mathrm{~h}$ of admission and Late WLS patients were those for whom a decision was made after $24 \mathrm{~h}$ of admission.

\subsection{Data analysis}

Baseline patient and injury characteristics were compared between groups (Alive/WLS/DLS) using Analysis of Variance for continuous variables and Pearson chi-squared of Fisher's exact test for discrete variables. To estimate the predictors of withdrawal of life support, we used a multivariate logistic regression model controlling for pertinent confounders (age, sex, race, TBSA, mechanism of injury, presence of inhalation injury and comorbidities using CCI).

Stata/MIP (version 15) (Statacorp, College Station, TX) was used for all data management and statistical analysis. A pvalue $<0.05$ was chosen to indicate statistical significance. This study was approved by the University of North Carolina, Institutional Review Board.

\section{Results}

During the study period, 11,977 patients were admitted to the UNC Jaycee Burn Center. 3483 patients <17years of age were 
excluded. An additional 124 patients were excluded including those in the DLS group $(n=109)$ and those missing discharge disposition $(n=15)$. The remaining 8371 patients were included for analysis.

Table 1 shows the baseline patient characteristics of this population. The majority of patients were male (72.4\%), white (56.7\%), and less than 65 years of age $(89.1 \%)$ with mean age 42.5years. Median CCI was 0. Median TBSA was 3\% (interquartile range [IQR] $1 \%-8 \%) .7 .1 \%$ of patients $(\mathrm{N}=591)$ suffered an inhalation injury and $9.9 \%$ of patients $(\mathrm{N}=828)$ were mechanically ventilated. A decision for WLS was made in 237 patients died during the study period.

Our bivariate analysis comparing WLS and Alive patients is shown in Table 2. There was a greater proportion of women in the WLS group than the Alive group ( $36.7 \%$ vs. $27.3 \%$ ) whereas as, there was a greater proportion of men in the Alive than WLS group $(72.7 \%$ vs. $63.3 \%)(p=0.001)$. WLS patients were older than Alive patients (41.9 years vs 62.4 years, respectively; $p<0.001$ ) and for both groups, the majority of patients were $<65$ years (WLS 51.5\% and Alive 90.2\%). Median CCI was 0 for both WLS and Alive patients $(p<0.001)$. Median TBSA was higher for WLS patients (WLS 31.5\% vs Alive 3\%; $<<0.001$ ). Median hospital LOS was longer in the Alive group (7days vs 5 days, respectively; $\mathrm{p}<0.001$ ). ICU LOS was greater for WLS patients (4 days vs 0 days, respectively; $\mathrm{p}<0.001$ ).

A multivariate logistic regression model was then performed, controlling for statistically significant covariates on

\section{Table 1 - Overall patient demographics and clinical} characteristics.

\begin{tabular}{ll} 
Overall $(\mathrm{N}=8371)$ & \multicolumn{1}{c}{$\mathrm{N} \%$} \\
Gender & \\
$\mathrm{M}$ & $6060(72.4)$ \\
$\mathrm{F}$ & $2311(27.6)$ \\
Age, overall (mean $\pm \mathrm{SD})$ & $42.5 \pm 16.7$ \\
Age, categorized & \\
$\quad$ 665years & $7455(89.1)$ \\
$\geq 65$ years & $915(10.9)$ \\
Unknown & $1(0.0)$ \\
Race & \\
White & $4747(56.7)$ \\
Black & $2371(28.3)$ \\
Hispanic & $598(7.1)$ \\
Other & $598(7.1)$ \\
Unknown & $56(0.7)$ \\
CCI, median (IQR) & $0(0)$ \\
Type of burn & \\
Flame & $4219(50.4)$ \\
Scald & $475(5.7)$ \\
Other & $3583(42.8)$ \\
Unknown & $94(1.1)$ \\
TBSA, median (IQR) & $3(1-8)$ \\
Inhalation injury & $591(7.1)$ \\
Unknown & $43(0.5)$ \\
Mechanical ventilation & $828(9.9)$ \\
Unknown & $238(2.8)$ \\
Hospital LOS, median (IQR) & $7(2-13)$ \\
ICU LOS, median (IQR) & $0(0-1)$ \\
WLS & $237(2.8)$ \\
WOC &
\end{tabular}

WOC=withdrawal of care; CCI=Charlson Comorbidity Index; $\mathrm{IQR}=$ interquartile range; LOS=length of stay.
Table 2 - Bivariate analysis comparing patient

demographics and characteristics for WLS and Alive.

\begin{tabular}{|c|c|c|c|}
\hline Characteristic & $\begin{array}{c}\text { WLS } \\
(\mathrm{N}=237)\end{array}$ & $\begin{array}{c}\text { Alive } \\
(\mathrm{N}=8134)\end{array}$ & p-Value \\
\hline \multicolumn{4}{|l|}{ Gender } \\
\hline M & $150(63.3)$ & $5910(72.7)$ & 0.001 \\
\hline $\mathrm{F}$ & $87(36.7)$ & $2224(27.3)$ & \\
\hline Age, overall & $41.9 \pm 16.3$ & $62.4 \pm 17.6$ & $<0.001$ \\
\hline Age, categorized & & & $<0.001$ \\
\hline$<65$ years & $122(51.5)$ & $7333(90.2)$ & \\
\hline$\geq 65$ years & $114(48.1)$ & $801(9.9)$ & \\
\hline Unknown & $1(0.4)$ & $0(0)$ & \\
\hline Race & & & 0.101 \\
\hline White & $141(59.5)$ & $4606(56.6)$ & \\
\hline Black & $63(26.6)$ & $2309(28.4)$ & \\
\hline Hispanic & $10(4.2)$ & $588(7.2)$ & \\
\hline Other & $19(8.0)$ & $579(7.1)$ & \\
\hline Unknown & $4(1.7)$ & $52(0.6)$ & \\
\hline CCI, median (IQR) & $0(0-2)$ & $0(0)$ & $<0.001$ \\
\hline Type of burn & & & $<0.001$ \\
\hline Flame & $209(88.2)$ & $4010(49.3)$ & \\
\hline Scald & $4(1.7)$ & $471(5.8)$ & \\
\hline Other & $20(8.4)$ & $3563(43.8)$ & \\
\hline Unknown & $4(1.7)$ & $90(1.1)$ & \\
\hline TBSA, median (IQR) & $31.5(9.3-64)$ & $3(1-7)$ & $<0.001$ \\
\hline Inhalation injury & $114(48.1)$ & $477(5.7)$ & $<0.001$ \\
\hline Unknown & $10(1.2)$ & $33(0.4)$ & \\
\hline Mechanical ventilation & $140(59.1)$ & $688(8.5)$ & $<0.001$ \\
\hline Unknown & $72(30.4)$ & $166(2.0)$ & \\
\hline Hospital LOS, median (IQR) & $5(1-41)$ & $7(2-13)$ & $<0.001$ \\
\hline ICU LOS, median (IQR) & $4(1-32)$ & $0(-1)$ & $<0.001$ \\
\hline
\end{tabular}

bivariate analysis and including other possible confounders: gender, age, race, burn etiology, CCI, \%TBSA, and inhalation injury (Table 3). Females had an increased odd of WLS compared to males (OR 2.03, 95\% CI 1.18-3.48; $\mathrm{p}=0.010$ )). Patients with associated comorbidities based on higher CCI had an increased odd of WLS (OR 1.28, 95\% CI 1.08-1.52; $\mathrm{p}=0.005)$. There was a significantly increased odd for WLS (OR

Table 3 - Multivariate logistic regression estimating risk of withdrawal of life support in a burn cohort.

$\begin{array}{lcl} & \begin{array}{c}\text { Adjusted OR, 95\% } \\ \text { Confidence interval (CI) }\end{array} & \text { p-Value } \\ \text { Gender (male ref) } & 2.03(1.18-3.48) & 0.010 \\ \text { Age, overall } & 1.09(1.06-1.12) & <0.001 \\ \text { Age, category } & & - \\ \quad \text { 65years (ref) } & - & 0.270 \\ \quad \text { 65years } & 00.61(0.25-1.48) & \\ \text { Race } & & - \\ \text { White (ref) } & - & 0.370 \\ \text { Black } & 0.77(0.44-1.36) & 0.454 \\ \text { Hispanic } & 0.49(0.08-3.18) & 0.731 \\ \text { Other } & 0.85(0.33-2.18) & - \\ \text { Etiology } & & 0.814 \\ \quad \text { Flame (ref) } & - & 0.456 \\ \quad \text { Scald } & 1.17(0.32-4.33) & 0.005 \\ \quad \text { Other } & 0.75(0.35-1.59) & <0.001 \\ \text { CCI } & 1.28(1.08-1.52) & <0.001 \\ \text { TBSA } & 1.08(1.07-1.51) & \\ \text { Inhalation injury } & 1.31(1.13-1.51) & \end{array}$

$\mathrm{OR}=$ odds ration; ref=reference . 
1.09, 95\% CI 1.06-1.12; $\mathrm{p}<0.001$ ) with increasing age. Similarly, there was an increased odd for WLS (OR 1.08, 95\% CI 1.07-1.51; $\mathrm{p}<0.001$ ) with increasing \%TBSA. An increased odd of WLS (OR $2.47,95 \%$ CI 1.05-5.78; $p=0.038$ ) was also found in patients with inhalation injury.

The majority of WLS patients (64.1\%) had support withdrawn $>24 \mathrm{~h}$ after admission $(\mathrm{n}=152)$ as noted in Table 4 . There were no statistical significant differences in patient characteristics between the Early and Late WLS cohort with respect to age $(64.5 \pm 18.7$ years vs $61.2 \pm 16.9$, respectively; $p=0.175)$ and median CCI (Early WLS 0 vs Late WLS $1 ; p=0.086$ ). TBSA was significantly greater in Early WLS than Late WLS patients (58.3\% vs $28 \%, p<0.001$ ) as was the presence of inhalation injury $(50.6 \%$ vs $46.7 \%, p=0.046)$. A greater percentage of patients were mechanically ventilated in the Late WLS group $(80.9 \%)$ than in the Early WLS $(20.0 \%)$ group $(p<0.001)$.

\section{Discussion}

The decision to withdraw life support on any patient is complex and difficult for all parties involved. While the death of a burn patient may occur following withdrawal of life support this decision must be informed by objective clinical data. In this study, we found that female gender and increasing age were significantly associated with an increased odd of WLS in our burn ICU (Table 5).

Quill et al. performed a review on decisions to forgo lifesustaining therapy (DFLST) for patients admitted to 153 ICUs (medical/surgical, cardiac care, trauma, neurologic) across the US [25]. They found that women were consistently more likely to have DFLSTs. They postulated that women are more likely to have less aggressive treatment preferences or to have surrogates that are more likely to make end of life decisions.

$\begin{array}{llll}\begin{array}{l}\text { Table 4 - Patient demographic and clinical characteristics } \\ \text { of the WLS group. }\end{array} & \begin{array}{c}\text { Early WLS } \\ (\mathrm{n}=85)\end{array} & \begin{array}{c}\text { Late WLS } \\ (\mathrm{n}=152)\end{array} & \mathrm{p} \text {-Value } \\ \text { Characteristic } & & & \\ \text { Gender } & 52(61.2) & 98(64.5) & 0.614 \\ \text { M } & 33(38.8) & 54(35.5) & \\ \text { F } & 64.5 \pm 18.7 & 61.2 \pm 16.9 & 0.175 \\ \text { Age, overall (mean } \pm \text { SD) } & & & 0.151 \\ \text { Race } & 45(52.9) & 96(63.2) & \\ \text { White } & 25(29.4) & 38(25.0) & \\ \text { Black } & 6(7.1) & 4(2.6) & \\ \text { Hispanic } & 6(7.1) & 13(8.6) & \\ \text { Other } & 3(3.5) & 1(0.7) & \\ \text { Unknown } & & & 0.073 \\ \text { Etiology } & 81(95.3) & 128(84.2) & \\ \text { Flame } & 0(0) & 4(2.6) & \\ \text { Scald } & 3(3.5) & 17(11.2) & \\ \text { Other } & 1(1.2) & 3(2.0) & \\ \text { Unknown } & 0(0-1) & 1(0-2) & 0.086 \\ \text { CCI, median (IQR) } & 58.3(1-82.5) & 28(12-50) & <0.001 \\ \text { TBSA, median (IQR) } & 43(50.6) & 71(46.7) & 0.046 \\ \text { Inhalation injury } & 7(8.2) & 3(2.0) & \\ \text { Unknown } & 17(20.0) & 123(80.9) & <0.001 \\ \text { Mechanical ventilation } & 60(70.6) & 12(7.9) & \\ \text { Unknown } & & & \\ & & & \end{array}$

Table 5-Multivariate logistic regression estimating odd of Late WLS.

$\begin{array}{lcc} & \begin{array}{c}\text { Adjusted OR, 95\% } \\ \text { Confidence interval (CI) }\end{array} & \text { p-Value } \\ \text { Gender (male ref) } & 1.67(0.64-4.37) & 0.299 \\ \text { Age, overall } & 0.98(0.94-1.03) & 0.411 \\ \text { Age, category } & & \\ \quad<65 \text { years (ref) } & - & - \\ \quad \text { 665years } & 0.95(0.19-4.60) & 0.946 \\ \text { Race } & & - \\ \quad \text { White (ref) } & - & 0.177 \\ \text { Black } & 2.20(0.70=6.94) & - \\ \text { Hispanic } & 1 & 0.452 \\ \text { Other } & 0.55(0.12-2.59) & - \\ \text { Etiology } & & - \\ \quad \text { Flame (ref) } & - & 0.340 \\ \text { Scald } & 1 & 0.098 \\ \text { Other } & 3.12(0.30-32.41) & 0.576 \\ \text { CCI } & 1.31(0.95-1.81) & 0.227 \\ \text { TBSA } & 1.00(0.99-1.02) & \\ \text { Inhalation injury } & 0.56(0.22-1.44) & \end{array}$

No clear data exists regarding how often women and men act as surrogate decision makers at the end of life. Women have been found, however, to serve as caregivers more often than men [26]. Health care surrogate decision making falls to the spouse by default in the absence of explicit and documented end of life wishes. As burn injury affects men more often than women and the life expectancy for women is longer one can assume that women will more often serve as surrogate decision makers than men. Further studies are needed to further elucidate the association of female sex with the end of life decision making process, especially among burn patients.

Other studies have previously shown that older age, increasing \%TBSA, and the presence of inhalation injury are significantly associated with an increased risk of death [7-10]. Furthermore, we showed that all were associated with increased likelihood for withdrawal of life support. Mahar et al. evaluated adult burn patients who died following withdrawal of life support and split them into Early WLS and Late WLS groups [27]. They noted there was no statistically significant differences between the two groups in terms of age, suggesting that age may not be a factor in the decision-making process as to the timing of WLS. We demonstrated similar findings in our subgroup analysis of WLS patients. Severity of burn was the only statistically significant patient characteristic between the two groups, suggesting that \%TBSA is the most important factor in early withdrawal of life support decisions.

In our study, the majority of WLS patients (64\%) were in the Late WLS group. Mechanical ventilation is often the final step in the withdrawal of life support process and has been shown to be significantly associated with higher daily costs for patients receiving care in the ICU $[8,28,29]$. Additionally, the extended length of stay of Late WLS patients drive up their total cost of care.

Defining futility in regard to the burn patient is challenging and the decision involves many factors that vary between institutions, patients and their families, as well as providers $[18,27,30-32]$. Recognition of which patients will most benefit 
from aggressive resuscitation and management is important and instituting a protocol can help in identifying these individuals. No set protocol was in place when Partain et al. evaluated Early end-of-life (EoL) discussions and Late EoL discussion in a cohort of geriatric burn patients [33]. Decisions for comfort care immediately after initial EoL discussions were similar between the groups. The early group had more severe injuries, shorter time to death, and fewer interventions, including operations.

Some institutions have begun implementing standardized withdrawal protocols and the early involvement of a palliative care team. Pham et al. noted that among patients who underwent life support withdrawal, there was an improvement in the number of patients who followed their institutional protocol after its implementation. There was also improved consistency in end-of-life symptoms palliation.

Few studies have evaluated the impact of preexisting medical comorbidities in burn injury outcomes [34-38]. A recent study from our institution demonstrated that preexisting comorbidities have a significant effect on burn mortality in all groups, particularly the younger age population [39]. In this current study this trend continues as we found that patients with comorbidities have a significant increase in odds of WLS. Ismail et al. evaluated 63 burn patients as to whether a Do-NotAttempt-Resuscitation (DNAR) order was issued and whether a decision to withdraw life-sustaining treatment was made [6]. Overall, a DNAR order and withdrawal of treatment took place in $33 \%$ of patients, while $27 \%$ of patients had withdrawal of treatment alone. They were able to evaluate patient charts for reasons why decisions were made. They noted that for patients $<65$ years, the decision was most commonly due to \%TBSA and burn depth while in the >65-year-old group, comorbidities were the main driver of withdrawal of life support. In the few papers that have evaluated reasons for WLS, none evaluated the effect of comorbidities [5,22]. For WLS patients, it is possible that the presence of comorbidities complicated their perceived clinical course, and therefore the decision to withdraw life support.

Limitations of this study include those inherent to a study with retrospective methodology. Furthermore, the design of this study limits our ability to fully explore the complexities the of end-of-life decision making process, including data regarding family as well as psychological data from those involved. Additionally, the decision for withdrawal of care varies between institutions, providers, and families. A patient's end-of-life wishes, as well as wishes from family members, play an important role in this decision and are difficult to measure. The definition of withdrawal of life support varies or may be interpreted by the health care providers in different ways. Lastly, defining futility for the burned injured patient is a dependent on burn specialist experience and local resources.

\section{Conclusions}

The decision to withdraw life support is a complex and difficult decision. Understanding the predictors of WLS will be indispensable to burn surgeons, caregivers and family members alike. At present, we have identified those factors that indicate an increased risk of mortality are likely to predict withdrawal of life support. Our findings, however, are limited given the study design. The role of patient's sex, particularly women, in WLS decision making needs to be further explored.

\section{Conflict of interest statement}

Predictors of Withdrawal of Life Support after Burn Injury.

The authors whose names are listed immediately below certify that they have NO affiliations with or involvement in any organization or entity with any financial interest (such as honoraria; educational grants; participation in speakers' bureaus; membership, employment, consultancies, stock ownership, or other equity interest; and expert testimony or patent-licensing arrangements), or non-financial interest (such as personal or professional relationships, affiliations, knowledge or beliefs) in the subject matter or materials discussed in this manuscript.

Author Names: Coleen Bartley MD, Kenisha Atwell MD, Laura Purcell MD, Bruce Cairns MD, Anthony Charles MD, MPH.

R E F E R E N C E S

[1] Burn Incidence Fact Sheet. https://ameriburn.org/who-weare/media/burn-incidence-fact-sheet/ [accessed on 2/8/18].

[2] National Fire Protection Association: Fire Loss in the U.S. during 2016. [accessed on 2/8/18, at https://www.nfpa.org/ News-and-Research/Fire-statistics-and-reports/Firestatistics/Fires-in-the-US/Overall-fire-problem/Fire-loss-inthe-United-States].

[3] Pham TN, Otto A, Young R, Kramer CB, Heimbach DM, Gibran NS, et al. Early withdrawal of life support in severe burn injury. J Burn Care Res 2012;33(January-February (1)):130-5, doi:http:// dx.doi.org/10.1097/BCR.0b013e31823e598d.

[4] Mahar PD, Wasiak J, Cleland H, Paul E, Loke SY, Fong HC, et al. Clinical differences between major burns patients deemed survivable and non-survivable on admission. Injury 2015;46 (May (5)):870-3, doi:http://dx.doi.org/10.1016/j. injury.2015.01.005.

[5] Ismail A, LongJ, Moiemen N, Wilson Y. End of life decisions and care of the adult burn patient. Burns 2011;37:288-93, doi:http:// dx.doi.org/10.1016/j.burns.2010.08.009

[6] O'Mara MS, Chapyak D, Greenhalgh DG, Palmieri TL. End of life in the pediatric burn patient. J Burn Care Res 2006;27 (November-December (6)):803-8.

[7] Cooke CR, Hotchkin DL, Engelberg RA, Rubinson L, Curtis JR. Chest 2010;138(August (2)):289-97, doi:http://dx.doi.org/ 10.1378/chest.10-0289.

[8] Hop MJ, Polinder S, Middelkoop E, van BM. Costs of burn care: A systematic review. Value Health 2014;17(November (7)):A606, doi:http://dx.doi.org/10.1016/j.jval.2014.08.2111.

[9] SánchezJL, Perepérez SB, Bastida JL, Martínez MM. Cost-utility analysis applied to the treatment of burn patients in a specialized center. Arch Surg 2007;142(January (1))7-50 discussion 57.

[10] Palmieri TL, Przkora R, Meyer 3rd WJ, Carrougher GJ. Measuring burn injury outcomes. Surg Clin North Am 2014;94 (August (4)):909-16, doi:http://dx.doi.org/10.1016/j. suc. 2014.05.010.

[11] Choinière M, Dumont M, Papillon J, Garrel DR. Prediction of death in patients with burns. Lancet 1999;353(Junuary (9171)):2211-2. 
[12] Kraft R, Herndon DN, Al-Mousawi AM, Williams FN, Finnerty CC, Jeschke MG. Burn size and survival probability in paediatric patients in modern burn care: a prospective observational cohort study. Lancet 2012;379(March (9820)):1013-21, doi:http://dx.doi.org/10.1016/S0140-6736(11) 61345-7.

[13] Jeschke MG, Pinto R, Kraft R, Nathens AB, Finnerty CC, Gamelli $\mathrm{RL}$, et al. Morbidity and survival probability in burn patients in modern burn care. Critical care medicine 2015;43(April (4)):808-15, doi:http://dx.doi.org/10.1097/ CCM.0000000000000790.

[14] Osler T, Glance LG, Hosmer DW. Simplified estimates of the probability of death after burn injuries: extending and updating the baux score. J Trauma 2010;68(March (3)):690-7, doi:http://dx.doi.org/10.1097/TA.0b013e3181c453b3.

[15] Heng JS, Clancy O, Atkins J, Leon-Villapalos J, Williams AJ Keays R, et al. Revised Baux Score and updated Charlson comorbidity index are independently associated with mortality in burns intensive care patients. Burns 2015;41 (November (7)):1420-7, doi:http://dx.doi.org/10.1016/j. burns.2015.06.009.

[16] Roberts G, Lloyd M, Parker M, Martin R, Philp B, Shelley O, et al. The Baux score is dead. Long live the Baux score: a 27-year retrospective cohort study of mortality at a regional burns service. J Trauma Acute Care Surg 2012;72(January (1)):251-6, doi:http://dx.doi.org/10.1097/TA.0b013e31824052bb.

[17] Tobiasen J, Hiebert JM, Edlich RF. The abbreviated burn severity index. Ann Emerg Med 1982;11(5):260-2.

[18] Ryan CM, Schoenfeld DA, Thorpe WP, Sheridan RL, Cassem EH, Tompkins RG. Objective estimates of the probability of death from burn injuries. N Engl J Med 1998;338(February (6)):362-6.

[19] Fisher M. Ethical issues in the intensive care unit. Curr Opin Crit Care 2004;10(August (4)):292-8, doi:http://dx.doi.org/ 10.1097/01.ccx.0000133670.35409.6a.

[20] Manara AR, Pittman JA, Braddon FE. Reasons for withdrawing treatment in patients receiving intensive care. Anaesthesia 1998;53(6):523, doi:http://dx.doi.org/10.1046/j. 1365-2044.1998. t01-1-00407. $\mathrm{x}$.

[21] Prendergast TJ, Claessens MT, Luce JM. A national survey of end-of-life care for critically ill patients. Am J Respir Crit Care Med 1998;158(4):1163-7, doi:http://dx.doi.org/10.1164/ ajrccm.158.4.9801108.

[22] Sprung CL, Cohen SL, Sjokvist P, Baras M, Bulow HH, Hovilehto $\mathrm{S}$, et al. End-of-life practices in European intensive care units: the Ethicus Study. JAMA 2003;290(August (6)):790-7, doi:http:// dx.doi.org/10.1001/jama.290.6.790.

[23] Ryan CM, Schoenfeld DA, Thorpe WP, Sheridan RL, Cassem EH, Tompkins RG. Objective estimates of the probability of death from burn injuries. N Engl J Med 1998;338(February (6)):362-6, doi:http://dx.doi.org/10.1056/NEJM199802053380604.

[24] Charlson ME, Pompei P, Ales L, MacKenzie CR. A new method of classifying prognostic comorbidity in longitudinal studies: development and validation. J Chronic Dis 1987;40:373-83.

[25] Quill CM, Ratcliffe SJ, Harhay MO, Halpern SD. Variation in decisions to forgo life-sustaining therapies in US ICUs. Chest 2014;146(September (3)):573-82, doi:http://dx.doi.org/10.1378/ chest.13-2529.
[26] Chappell NL, Dujela C, Smith A. Caregiver Well-Being. Intersections of Relationship and Gender. Res Aging 2015;37 (August (6)):623-45, doi:http://dx.doi.org/10.1177/ 0164027514549258 .

[27] Mahar PD, Wasiak J, Cleland H, Paul E, Loke SY, Fong HC, et al. Clinical differences between major burns patients deemed survivable and non-survivable on admission. Injury 2015;46 (May (5)):870-3, doi:http://dx.doi.org/10.1016/j. injury.2015.01.005.

[28] Long AC, Muni S, Treece PD, Engelberg RA, Nielsen EL, Fitzpatrick AL, et al. Time to death after terminal withdrawal of mechanical ventilation: specific respiratory and physiologic parameters may inform physician predictions. J Palliat Med 2015;18(December (12)):1040-7, doi:http://dx.doi. org/10.1089/jpm.2015.0115.

[29] Dasta JF, McLaughlin TP, Mody SH, Piech CT. Daily cost of an intensive care unit day: the contribution of mechanical ventilation. Crit Care Med 2005;33(June (6)):1266-71.

[30] den Hollander D. Medical futility and the burns patient. Burns 2013;39(August (5)):851-5, doi:http://dx.doi.org/10.1016/j. burns.2013.02.001

[31] Fratianne RB, Brandt CP. Determining when care for burns is futile. J Burn Care Rehabil 1997;18(May-June (3))262-7 discussion 260-1.

[32] Metaxa V, Lavrentieva A. End-of-life decisions in burn intensive care units - an international survey. Burns 2015;41 (February (1)):53-7, doi:http://dx.doi.org/10.1016/j. burns.2014.05.018.

[33] Partain NS, Subramanian M, Hodgman EI, Isbell CL, Wolf SE, Arnoldo BD, et al. Characterizing end of life care after geriatric burns at a verified level I burn center. J Palliat Med 2016;19 (December (12)):1275-80, doi:http://dx.doi.org/10.1089/ jpm.2016.0152.

[34] Thombs BD, Singh VA, Halonen J, Diallo A, Milner SM. The effects of preexisting medical comorbidities on morality and length of hospital stay in acute burn injury: evidence from a national sample of 31,338 adult patients. Ann Surg 2007;245 (April (4)):629-34, doi:http://dx.doi.org/10.1097/01. sla.0000250422.36168.67.

[35] Lundgren RS, Kramer CB, Rivara FP, Wang J, Heimbach DM, Gibran NS, et al. Influence of comorbidities and age on outcome following burn injury in older adults. J Burn Care Res 2009;30(March-April (2)):307-14, doi:http://dx.doi.org/10.1097/ BCR.0b013e318198a416

[36] Germann G, Barthold U, Lefering R, Raff T, Hartmann B. The impact of risk factors and pre-existing conditions on the mortality of burn patients and the precision of predictive admission-scoring systems. Burns 1997;23(May (3)):195-203.

[37] Covington DS, Wainwright DJ, Parks DH. Prognostic indicators in the elderly patient with burns. J Burn Care Rehabil 1996;17 (May-June (3)):222-30.

[38] Raff T, Germann G, Barthold U. Factors influencing the early prediction of outcome from burns. Acta Chir Plast 1996;38 (4):122-7.

[39] Knowlin L, Stanford L, Moore D, Cairns B, Charles A. The measured effect of co-morbidities on burn injury mortality. Burns 2016;42(November (7))1433-8, doi:http://dx.doi.org/ 10.1016/j.burns.2016.03.007 Epub 2016 Sep 1. 